\title{
APROVISIONAMIENTO Y ANÁLISIS DE SEMILLAS DE HUALO PARA SU USO EN PROYECTO FIBN 002/2018: SIEMBRA DIRECTA: TÉCNICA DE RECUPERACIÓN DE BOSQUES NATIVOS DE ROBLE Y HUALO
}

Gutiérrez, Braulio; Quiroz, Iván y Koch, Laura ${ }^{16}$

\section{RESUMEN}

Se evalúa el efecto del remojo por 24 horas en ácido giberélico $(0,50,100,200$ y 400 ppm) sobre la germinación de semillas de hualo (Nothofagus glauca) de dos localidades de la región del Maule (Quivolgo y Empedrado). En ambos casos la germinación de los tratamientos es significativamente superior a la del testigo, obteniéndose la mejor germinación con la mayor concentración de ácido giberélico. En Empedrado se logra una germinación máxima de 26,7\% y en Quivolgo 9,3\% atribuyéndose la diferencia a la calidad de cada lote de semillas. En efecto la semilla de Quivolgo es de menor tamaño (3.243 sem $/ \mathrm{kg}$ contra $1.924 \mathrm{sem} / \mathrm{kg}$ de Empedrado) y con mayor incidencia de perforaciones que afectan su viabilidad.

Palabras clave: Nothofagus glauca, semillas, germinación.

\section{SUMMARY}

The effect of soaking for 24 hours in gibberellic acid $(0,50,100,200$ and $400 \mathrm{ppm})$ on the germination of hualo seeds (Nothofagus glauca) from two localities of the Maule region (Quivolgo and Empedrado) is evaluated. In both cases the germination of the treatments is significantly superior to control without treatment, obtaining the best germination with the highest concentration of gibberellic acid. In Empedrado the maximum germination was $26.7 \%$ meanwhile in Quivolgo was $9.3 \%$; this difference was attributed to the quality of each seed lot. In fact, the Quivolgo seed is smaller (3,243 seeds/kg versus 1,924 seeds $/ \mathrm{kg}$ at Empedrado) and with a higher incidence of perforations that affect its viability.

Keywords: Nothofagus glauca, seeds, germination

${ }^{16}$ Investigadores, Instituto Forestal, Chile, Sede Bio Bio bgutierr@infor.cl 


\section{INTRODUCCIÓN}

El hualo (Nothofagus glauca (Phil.) Krasser) es una especie endémica característica de los bosques mediterráneos o mesomórficos de Chile central, donde crece en forma natural en las altitudes medias de las cordilleras de Los Andes (bajo los $1.200 \mathrm{msnm}$ ) y de la Costa (150 a 800 msnm), entre las provincias de Cachapoal y del Bio Bio (MMA, s/f). La máxima concentración de esta especie se encuentra en la costa de las provincias de Talca y Cauquenes (Región del Maule), donde forma masas continuas de importancia.

Las poblaciones de hualo han sido profundamente afectadas por la intervención humana. Sus bosques han sufrido fuerte presión antrópica, tanto por el desmonte del bosque natural para la habilitación del suelo para otros usos como por la explotación de sus renovales para la obtención de leña y carbón, encontrándose en la categoría de especie casi amenazada (MMA s/f). En sus áreas de mayor abundancia en la costa de la región del Maule sus poblaciones resultaron severamente afectadas por los agrandes incendios forestales del año 2017. En ese contexto, en el proyecto FIBN 002/2018 "Siembra Directa: Técnica de Recuperación de Bosques Nativos de Roble y Hualo", se ha propuesto evaluar la pertinencia de la siembra directa como mecanismo para recuperar estos bosques quemados.

Consecuentemente, en el presente informe se entregan los resultados de aprovisionamiento de semillas, así como la evaluación de las mismas en términos físicos y de germinación, de modo de contar con los antecedentes básicos para dimensionar los requerimientos de material para las labores de siembra directa y de producción de plantas involucrados en el mencionado proyecto.

\section{MATERIAL Y MÉTODO}

\section{Aprovisionamiento y Análisis de Semillas}

Se dispuso de 2 lotes de semillas de hualo colectados en marzo del año 2019 desde rodales naturales de la zona de Empedrado y de Quivolgo. La colecta fue realizada por contratistas de las empresas forestales asociadas al proyecto, como parte de su aporte al mismo.

Recibidos los sacos de semillas, estas fueron trasladadas a dependencias de INFOR Bio Bio, donde se procedió a realizar los respectivos análisis físicos (\%pureza y número de semillas por $\mathrm{Kg}$ ) y de germinación (\%capacidad germinativa; \%energía germinativa y periodo de energía en días), mediante pruebas experimentales orientadas por los lineamientos de la asociación internacional de análisis e semillas (ISTA, 2016).

\section{Determinación del Porcentaje de Pureza}

Se tomaron tres muestras de semillas de al menos $200 \mathrm{~g}$ cada una; se pesó cada muestra en una balanza analítica; se limpió manualmente cada una de las muestras, removiendo restos de hoja, tierra, ramillas y otras impurezas; se pesó la semilla limpia y se estimó el porcentaje de pureza con la fórmula 1.

$$
\text { Pureza }(\%)=(\text { Peso semilla limpia/ Peso muestra }) \times 100
$$

\section{Determinación del Número de Semillas por Kilogramo}

Esta determinación se efectuó con las mismas muestras de semilla limpia obtenidas en el análisis anterior; se contó el número de semillas contenidas en cada muestra y se estimó el número de unidades por kilogramo utilizando la fórmula 2. 
$\mathrm{N}^{\circ}$ sem por $\mathrm{Kg}=1.000 \times\left(\mathrm{N}^{\circ}\right.$ sem en la muestra/peso de la muestra en gramos $)(2)$

\section{Germinación}

Se montó un ensayo de germinación en el que fueron comparados cuatro tratamientos pregerminativos y un control sin tratamiento, los tratamientos ensayados (Cuadro $\mathrm{N}^{\circ} 1$ ) correspondieron a remojo por 24 horas en soluciones de ácido giberélico de distinta concentración.

Previo a la aplicación de los tratamientos pregerminativos, las semillas fueron desinfectadas mediante remojo en solución de hipoclorito de sodio al $10 \%$ durante 5 minutos y luego enjuagadas tres veces en agua destilada estéril.

Cuadro $\mathrm{N}^{\circ} 1$

TRATAMIENTOS PREGERMINATIVOS APLICADOS

\begin{tabular}{|c|c|c|c|}
\hline Tratamientos & Remojo & $\begin{array}{c}\text { Concentración Ácido } \\
\text { Giberélico } \\
\text { (ppm) }\end{array}$ & $\begin{array}{l}\text { Tiempo } \\
\text { (h) }\end{array}$ \\
\hline T0 (testigo) & Agua & $0 \mathrm{ppm}$ & \multirow{5}{*}{$24 \mathrm{~h}$} \\
\hline $\mathrm{T} 1$ & \multirow{4}{*}{ Solución de ácido giberélico } & 50ppm & \\
\hline T2 & & 100 ppm & \\
\hline T3 & & 200 ppm & \\
\hline T4 & & $400 \mathrm{ppm}$ & \\
\hline
\end{tabular}

El testigo y cada uno de los tratamientos fueron representados por tres repeticiones de 50 semillas cada una, dispuestas en placas Petri con papel filtro húmedo e incubadas en cámara germinadora, en oscuridad y a una temperatura de $22^{\circ} \mathrm{C}$. (Figura $\mathrm{N}^{\circ} 1$ ).
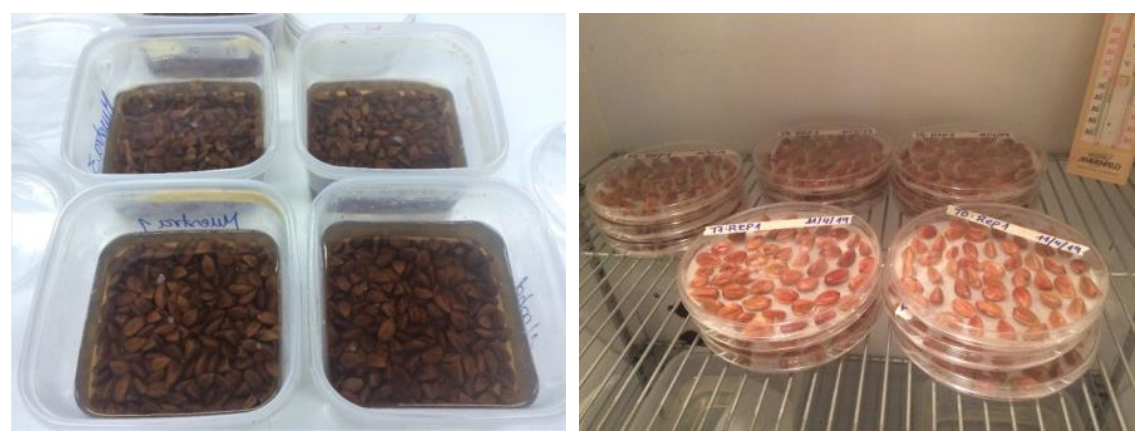

Figura $\mathrm{N}^{\circ} 1$

REMOJO SEMILLAS EN SOLUCIONES CON DISTINTAS CONCENTRACIONES DE ÁCIDO GIBERÉLICO (Izq). VISTA DE LAS PLACAS PETRI DISPUESTAS EN CAMARA GERMINADORA (Der.)

El ensayo fue monitoreado hasta que se detuvo la germinación, registrándose diariamente el número de semillas germinadas en cada repetición de cada tratamiento, y retirándolas de las placas. 
Como criterio de germinación se asumió la presencia de radícula extendida en una longitud mayor a $5 \mathrm{~mm}$. El número de semillas germinadas por día se expresó como porcentaje de germinación acumulada diaria, para posteriormente determinar los parámetros: Capacidad Germinativa (CG\%); Energía Germinativa (EG\%) y Periodo de Energía (PE) de cuerdo a la metodología tradicional del valor máximo de Czabator.

Se realizó análisis de varianza y pruebas de comparación de medias con alfa de 0,05 para determinar la existencia de diferencias estadísticamente significativas entre tratamientos.

Para estos análisis los valores en porcentaje de capacidad y energía germinativa fueron previamente transformados a unidades de Bliss (transformación de arco seno).

\section{RESULTADOS Y DISCUSIÓN}

En total se dispuso $51,9 \mathrm{~kg}$ de semillas de la procedencia Empedrado y de una cantidad significativamente menor de la procedencia Quivolgo (alrededor de $5 \mathrm{Kg}$ ). Los resultados de la caracterización de ambos lotes se detallan a continuación.

\section{Parámetros Físicos}

Ambos lotes, Empedrado y Quivolgo, presentan una alta pureza, cercana al 100\%, dado que son semillas grandes y por lo mismo relativamente fáciles de limpiar (Cuadro $\mathrm{N}^{\circ} 2$ ).

En cuanto al número de semillas por kilo, este resulta considerablemente menor en el lote de Empedrado $(1.924 \mathrm{sem} / \mathrm{kg})$, denotando un mayor tamaño de semillas que las del lote de Quivolgo (3.243 sem/kg) (Cuadro $\mathrm{N}^{\circ} 3$ ).

Cuadro $\mathrm{N}^{\circ} 2$

DETERMINACIÓN DE PORCENTAJE DE PUREZA

\begin{tabular}{|l|l|r|r|r|c|}
\hline Lote & & Muestra 1 & Muestra 2 & Muestra 3 & \multirow{2}{*}{ Pureza } \\
\hline \multirow{3}{*}{ Empedrado } & Peso muestra (g) & 200,34 & 200,53 & 200,52 & \multirow{3}{*}{$\mathbf{9 9 , 5}$} \\
\cline { 2 - 5 } & Peso semilla limpia (g) & 199,21 & 199,37 & 199,96 & \\
\cline { 2 - 4 } & Pureza (\%) & $\mathbf{9 9 , 5}$ & $\mathbf{9 9 , 4}$ & $\mathbf{9 9 , 7}$ & \\
\hline \multirow{3}{*}{ Quivolgo } & Peso muestra (g) & 200,28 & 200,20 & 200,24 & \multirow{3}{*}{$\mathbf{9 9 , 9}$} \\
\cline { 2 - 4 } & Peso semilla limpia (g) & 200,21 & 200,05 & 200,18 & \\
\cline { 2 - 4 } & Pureza (\%) & $\mathbf{9 9 , 9}$ & $\mathbf{9 9 , 9}$ & $\mathbf{9 9 , 9}$ & \\
\hline
\end{tabular}

Cuadro $\mathrm{N}^{\circ} 3$

DETERMINACIÓN DE NÚMERO DE SEMILLAS POR KILOGRAMO

\begin{tabular}{|c|c|c|c|c|c|}
\hline Lote & & Muestra 1 & Muestra 2 & Muestra 3 & ( sem/kg) \\
\hline \multirow{3}{*}{ Empedrado } & Peso semilla limpia (g) & 199 & 199 & 200 & \multirow{3}{*}{1.924} \\
\hline & $\mathrm{N}^{\circ}$ de semillas en la muestra & 380 & 382 & 389 & \\
\hline & $\mathrm{N}^{\circ}$ de semillas por $\mathrm{Kg}$ & 1909 & 1919 & 1945 & \\
\hline \multirow{3}{*}{ Quivolgo } & Peso semilla limpia $(\mathrm{g})$ & 200 & 200 & 200 & \multirow{3}{*}{3.243} \\
\hline & $\mathrm{N}^{\circ}$ de semillas en la muestra & 641 & 641 & 665 & \\
\hline & $\mathrm{N}^{\circ}$ de semillas por $\mathrm{Kg}$ & 3.202 & 3.204 & 3.322 & \\
\hline
\end{tabular}

El valor obtenido de número de semillas por kilogramo es algo mayor al indicado por Santelices et al. (1996), indicando que las semillas serían de menor tamaño que las mencionadas por esos autores para procedencias costeras de hualo (1500 semillas $/ \mathrm{kg}$ ). 
En cualquier caso, el número de semillas por kilogramo es un parámetro que suele ser muy variable entre procedencias, árboles y temporadas de semillación, pudiendo fluctuar ampliamente en hualo entre 1.200 y 3.800 unidades por kilo (Muñoz, 1993).

En cuanto a pureza, el lote evaluado da valores superiores a los mencionados por Santelices et al. (1996), quienes indican porcentajes entre 63 y $79 \%$.

\section{Parámetros de Germinación}

En el lote Empedrado la evaluación de la germinación evidenció una respuesta proporcional a la dosis de ácido giberélico utilizada. La capacidad germinativa entendida como la germinación observada al final del ensayo alcanzó a $26,67 \%$ en el tratamiento 4 (400 ppm); la energía germinativa, que corresponde a la máxima tasa o velocidad de germinación la obtuvo el tratamiento $2(18,67 \%)$; mientras que el menor periodo de energía, o tiempo requerido para obtener la máxima tasa de germinación, correspondió también al tratamiento 4 con 13 días. Por su parte, el testigo sin aplicación de ácido giberélico obtuvo la menor germinación $(8,67 \%)$, menor tasa de germinación y el mayor periodo de energía (30 días, germinación más lenta).

El resumen de estos resultados, junto con la diferenciación estadística, se presenta en el Cuadro $N^{\circ} 4$. La evolución de la germinación acumulada diaria para el testigo y los diferentes tratamientos ensayados se indica en la Figura $\mathrm{N}^{\circ} 2$.

La directa relación entre germinación y concentración de ácido giberélico indica que es posible seguir mejorando la germinación usando concentraciones superiores a 400 ppm, sugiriéndose nuevos ensayos para determinar los umbrales de hormona antes de que se haga tóxica o detrimental para la germinación.

\section{Cuadro $\mathrm{N}^{\circ} 4$}

PARÁMETROS DE GERMINACIÓN POR TRATAMIENTO LOTE EMPEDRADO

\begin{tabular}{|c|r|r|r|}
\hline Tratamiento & \multicolumn{1}{|c|}{$\begin{array}{c}\text { Capacidad } \\
\text { Germinativa } \\
\text { (\%) }\end{array}$} & $\begin{array}{c}\text { Energía } \\
\text { Germinativa } \\
\text { (\%) }\end{array}$ & \multicolumn{2}{|c|}{$\begin{array}{c}\text { Periodo de } \\
\text { Energía } \\
\text { (días) }\end{array}$} \\
\hline T4 & $26,67 \mathrm{a}$ & $15,33 \mathrm{a}$ & $13 \mathrm{a}$ \\
\hline T3 & $25,33 \mathrm{a}$ & $18,00 \mathrm{a}$ & $17 \mathrm{a}$ \\
\hline T2 & $21,33 \mathrm{a}$ & $18,67 \mathrm{a}$ & $24 \mathrm{~b}$ \\
\hline T1 & $14,67 \mathrm{~b}$ & $14,00 \mathrm{a}$ & $30 \mathrm{~b}$ \\
\hline T0 & $8,67 \mathrm{~b}$ & $8,67 \mathrm{~b}$ & $\mathrm{a}$ \\
\hline
\end{tabular}

Letras distintas en una misma columna indican diferencias estadísticamente significativas entre medias (alfa $=0,05)$

En su conjunto, el lote Quivolgo presentó una germinación significativamente menor que la observada en Empedrado, situación que se refleja en el Cuadro $\mathrm{N}^{\circ} 5$ y Figura $\mathrm{N}^{\circ} 2$. El mejor tratamiento continúa siendo T4, que involucra la mayor dosis de ácido giberélico, pero en este caso alcanza solo a un 9,3\% de germinación, en contraste con el 26,7 conseguido en el lote Empedrado.

La baja germinación del tratamiento T4 de Quivolgo resulta equivalente a la del testigo sin tratamiento del lote Empedrado. 
Cuadro $\mathrm{N}^{\circ} 5$

PARÁMETROS DE GERMINACIÓN POR TRATAMIENTO LOTE QUIVOLGO

\begin{tabular}{|c|r|r|c|}
\hline Tratamiento & \multicolumn{1}{|c|}{$\begin{array}{c}\text { Capacidad } \\
\text { Germinativa } \\
\text { (\%) }\end{array}$} & $\begin{array}{c}\text { Energía } \\
\text { Germinativa } \\
\text { (\%) }\end{array}$ & $\begin{array}{c}\text { Periodo de } \\
\text { Energía } \\
\text { (días) }\end{array}$ \\
\hline T4 & 9,33 & 8,67 & 16 \\
\hline T3 & 4,00 & 4,00 & 18 \\
\hline T2 & 8,00 & 6,67 & 14 \\
\hline T1 & 6,67 & 6,67 & 20 \\
\hline T0 & 0,67 & 0,67 & 24 \\
\hline
\end{tabular}

Por otra parte, en el lote Quivolgo se observa la misma tendencia a que los resultados de germinación se relacionen directamente con la concentración de hormona utilizada en el tratamiento pregerminativo, aunque en este caso el resultado obtenido por el tratamiento T3 es anómalo, solo supera al testigo y es menor al obtenido por los tratamientos T1 y T2.
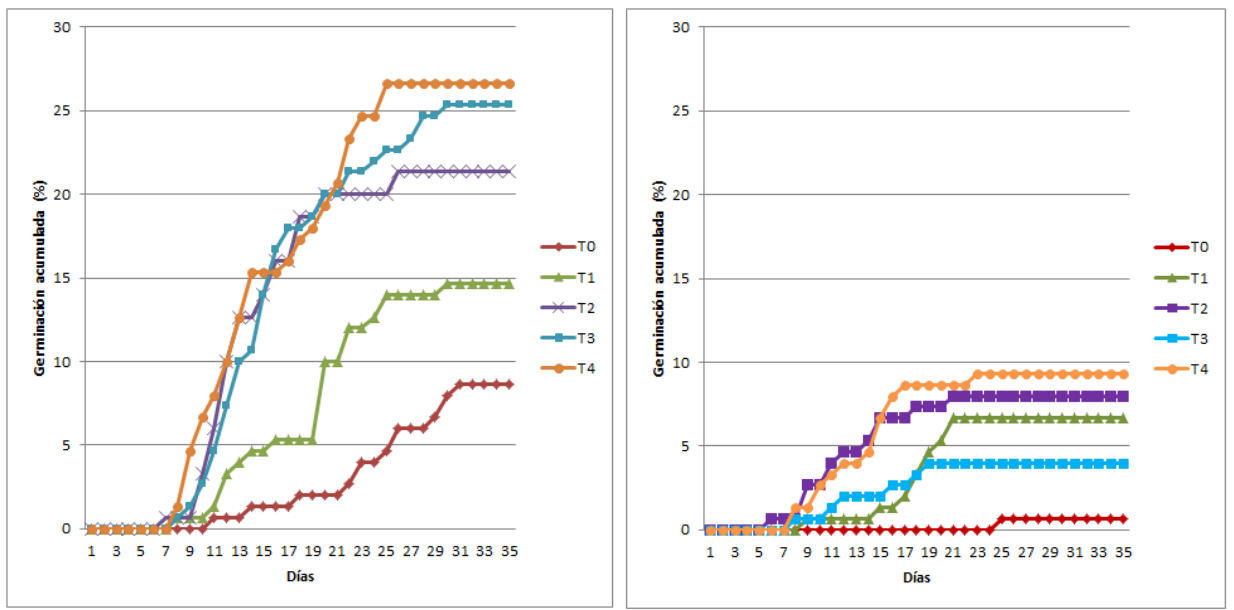

Figura $\mathrm{N}^{\circ} 2$

GERMINACIÓN ACUMULADA DIARIA POR TRATAMIENTO LOTE EMPEDRADO (izq.) LOTE QUIVOLGO (der.)

En cualquier caso, la germinación observada en Quivolgo resulta atribuible a las características del lote de semillas más que a los tratamientos pregerminativos, por cuanto al comparar los resultados de los testigos de ambos lotes, empedrado obtiene cerca de un $9 \%$ mientras que Quivolgo no alcanza al 1\%. En efecto, la semilla del lote Quivolgo es mucho más pequeña que la de Empedrado, situación reflejada en el número de semillas por kilo, y como indica Escobar (2007) generalmente las semillas más pequeñas de una muestra tienen menor viabilidad y mayor latencia que las intermedias y más grandes. 
Adicionalmente, debe destacarse que, en el caso de las semillas colectadas en Empedrado, atendiendo al gran volumen cosechado, el contratista encargado de la faena efectuó una rigurosa selección de las semillas, aspecto que no fue considerado en el caso de las semillas colectadas en Quivolgo, por cuanto la cosecha resultó muy escasa.

Las semillas de hualo, como las de la mayoría de los Nothofagus, presentan latencia fisiológica y requieren de tratamientos pregerminativos para acelerar y aumentar su germinación. Los tratamientos comúnmente utilizados para estos efectos consisten en estratificación fría por periodos variables, entre 4 a 6 semanas, y remojos en soluciones estimulantes como ácido giberélico o tiourea.

Al respecto, Espinoza y Cabello (1993) señalan que remojos en ácido giberélico entre 25 y 800 ppm producirían germinación de entre 84 y 97\%, destacando que este tipo de remojos es más efectivo que los tratamientos de estratificación fría a $5^{\circ} \mathrm{C}$ por 30 a 60 días, que según su duración puede producir germinación de 72 a 95\%; por su parte los tratamientos en base a tiourea tiene resultados más variables, entre 5 y 90\%. Confirmando lo anterior, Cabello (1995, cit. por Santelices et al., 1996) indica que los remojos en tiourea arrojan resultados muy variables, que dependiendo de la concentración $(0,75 \%$ a $3 \%)$ pueden fluctuar entre 92 y $3 \%$ de germinación.

De entre los tratamientos mencionados, el remojo en ácido giberélico es una práctica frecuente para reemplazar los extensos periodos involucrados en los procesos de estratificación fría, siendo normal utilizar concentraciones cercanas a los 200 ppm para especies de Nothofagus (Medina, 2000).

Los antecedentes reflejan porcentajes de germinación para hualo sustantivamente superiores a los obtenidos en este ensayo. No obstante, ensayos efectuados por Santelices et al. (1996) con semilla de hualo de procedencias costeras, obtienen germinación de 37 a $56 \%$ con estratificación de 30 y 60 días respectivamente; de $20 \%$ con remojo en tiourea ( $0,5 \%$ por 48 horas); y no más del $2 \%$ en los testigos.

\section{REFERENCIAS}

Escobar, R., 2007. Manual de viverización. Instituto Forestal, Centro Tecnológico de la Planta Forestal. Concepción, Chile. 230 p.

Espinoza, N. y Cabello, A., 1993. Propagación del hualo (Nothofagus glauca) por semillas. En: Avances en silvicultura. Universidad de Chile. Facultad de Ciencias Agrarias y Forestales. Departamento de Silvicultura. Publicaciones Misceláneas Forestales N¹. Santiago. Chile. 23p.

ISTA, 2016. Reglas internacionales para el análisis de las semillas. Introducción a las reglas ISTA Capítulos 1-7, 9. International Seed Testing Asociation (ISTA), Zurichstr. Basserrsdorf, Suiza. Online ISSN 2310-3655.

Medina, A., 2000. Viverización de procedencias de Nothofgus obliqua y N. alpina en contenedores. En: Ipinza, R.; Gutiérrez, B y Emhart, V (Eds.). Domesticación y Mejora Genética de Roble y Raulí. Instituto Forestal Universidad Austral de Chile. Pp: 307-322.

MMA. s/f. Ficha de especie Nothofagus glauca (Phil.) Krasser. Ministerio del medioambiente, Chile. 8 p.

Muñoz, M., 1993. Algunos antecedentes sobre propagación de Nothofagus. Ciencia e Investigación Forestal 7(2):377-389.

Santelices, R.; Riquelme, M. y Rojas, F., 1996. Aspectos sobre la semilla y germinación de Nothofagus glauca (Phil.) Krasser de dos procedencias de la VII región. Ciencia e Investigación Forestal 10(2):297-305. 
